\title{
Adaptação e validação brasileira do instrumento de avaliação do luto prolongado - PG-I3
}

\begin{abstract}
Mayra Delalibera'
Instituto Superior de Psicologia Aplicada - ISPA, Lisboa, Portugal
\end{abstract}

Talia Armani Delalibera

Hospital Santa Marcelina, SP, Brasil

Maria Helena Pereira Franco

Pontifícia Universidade Católica de São Paulo, SP, Brasil

António Barbosa

Faculdade de Medicina da Universidade de Lisboa, Lisboa, Portugal

Isabel Leal

Instituto Superior de Psicologia Aplicada - ISPA, Lisboa, Portugal

\begin{abstract}
Resumo: O luto pode ser definido como um conjunto de reações emocionais, físicas, comportamentais e sociais que surgem como resposta a uma perda significativa. $\mathrm{O}$ objetivo do estudo foi traduzir, adaptar e validar para a população brasileira o instrumento de avaliação do luto prolongado PG-13. A amostra foi constituída por II5 familiares enlutados que preencheram um questionário on-line. Desses familiares, $76,5 \%$ eram do sexo feminino, 50,4\% casados, idade média de 41,32 anos, e com tempo médio de luto de 29,29 meses. A incidência de luto prolongado foi de $10,43 \%$. Todos os itens do modelo reestruturado do PG- I 3 apresentaram pesos fatoriais superiores a 0,5 , eram estatisticamente significativos e com confiabilidades individuais adequadas. A consistência interna foi de 0,940 e a validade convergente avaliada pela VEM foi de 0,593 . É possível concluir que o PG-13 apresenta confiabilidade individual, de construto e validade convergente, podendo assim contribuir para o diagnóstico do luto prolongado.
\end{abstract}

Palavras-chave: estudos de validação; psicometria; luto; luto prolongado; PG-I3.

\section{ADAPTATION AND VALIDATION FOR THE BRAZILIAN POPULATION TO ASSESS- MENT INSTRUMENT PROLONGED GRIEF DISORDER - PG-13}

\begin{abstract}
Grief may be defined as a set of emotional, physical, behavioral, and social reactions that appear in response to a significant loss. The study aims at translating, adapting and validating, for the Brazilian population, the assessment tool of prolonged grief PG-13. The sample consisted of II 5 bereaved family members who completed a questionnaire online. Of these families, $76,5 \%$ were female, $50,4 \%$ were married, mean age of 4I,32 years and a mean time of grief 29,29 months. The incidence of prolonged grief was $10,43 \%$. All items on the restructured model PG-I3 showed higher factor weights 0,5 , they were statistically significant and had appropriate individual reliabilities. The Cronbach's alpha was 0,940 and the convergent validity assessed by VEM was 0,593 . Therefore, we conclude that the PG-I 3 has individual and construct reliability and convergent validity that could also contribute to the diagnosis of prolonged grief.
\end{abstract}

Keywords: validation studies; psychometry; grief; prolonged grief; PG-I3.

1 Endereço de correspondência: Mayra Delalibera; Rua Dom Afonso Henriques, 217 - $8^{\circ}$ Ai; Estoril - Portugal 2765-571 - Telefone (351) 917-383-386. E-mail: mayrarmani@yahoo.com.br. 
ADAPTACIÓN Y VALIDACIÓN PARA LA POBLACIÓN BRASILEÑA LA HERRAMIENTA

DE EVALUACIÓN DE DUELO PROLONGADO - PG-13

\begin{abstract}
Resumen: El duelo puede ser definido como un conjunto de reacciones emocionales, físicas, conductuales y sociales que surgen en respuesta a una pérdida significativa. El objetivo de este estudio fue traducir, adaptar y validar para la población brasileña la herramienta de evaluación de duelo prolongado PG-13. La muestra está formada por I I 5 familiares en duelo que han cumplimentado el cuestionario en línea. De estas familias, el $76,5 \%$ eran mujeres, el $50,4 \%$ estaban casadas, la edad media de $4 I, 32$ años y con una media de 29,29 meses de duelo. La incidencia de duelo prolongado fue 10,43\%. Todos los elementos del modelo reestructurado PG-13 mostraron pesos factoriales superiores 0.5 , fueron estadísticamente significativos y con fiabilidades individuales adecuados. El alfa de Cronbach fue de 0,940 y la validez convergente evaluado por VEM fue 0,593. Se concluyó que PG-I 3 tiene fiabilidad individual, de constructo y validez convergente, que contribuyen al diagnóstico de duelo prolongado.
\end{abstract}

Palabras-claves: estudios de validación; psicometría; duelo; duelo prolongado; PG-I3.

\title{
Introdução
}

O luto é uma resposta universal e natural à perda de um ente querido, sendo esse um acontecimento estressante que a maioria das pessoas terá que enfrentar ao longo da vida. O luto pode ser definido como um conjunto de reações emocionais, físicas, comportamentais e sociais que aparecem como resposta a uma perda significativa (Parkes, 1998).

Diante da perda de um ente querido, a maioria das pessoas desenvolve um processo de luto normal, superando os sentimentos de descrença, desespero e dor, e gradualmente se tornam capazes de encarar a realidade da perda e retomar os seus interesses, atividades de vida diária e o trabalho (Prigerson, 2004; Rando, 2013). No entanto, uma parcela de $10 \%$ a $20 \%$ da população enlutada tem dificuldades em lidar com a perda e pode desenvolver sintomas debilitantes de ordem física, mental e comportamental e incapacidade funcional persistente (Boerner, Mancini, \& Bonanno, 2013; Boelen \& Prigerson, 2007; Prigerson, Vanderwerker, \& Maciejewski, 2008; Prigerson, 2004). Sabe-se atualmente que, entre os extremos de uma reação de luto adaptada, não merecedora de intervenção especializada, e as síndromes psiquiátricas que podem se desenvolver na sequência de uma perda, há uma população intermediária de indivíduos que apresentará complicações no processo de luto, cuja severidade e duração dos sintomas justificam intervenção terapêutica (Prigerson et al., 1995, 1997, 1999; Prigerson, 2004).

Estudos têm demonstrado que o luto prolongado afeta a qualidade de vida e a saúde mental dos indivíduos (Boelen \& Prigerson, 2007), resultando, de acordo com Parkes (1998), em um aumento do número de consultas médicas, hospitalizações, cirurgias e taxa de mortalidade na população de enlutados, quando comparado à população geral. O luto complicado também está associado à depressão, à ansiedade, ao abuso de álcool e de medicamentos, ao maior risco de doenças e suicídios (Prigerson et al., 1997; Prigerson, 2004).

É importante esclarecer que o transtorno de luto prolongado é distinto e apresenta sintomatologia diferente de outros transtornos psiquiátricos, como o transtorno de 
estresse pós-traumático, transtorno depressivo maior, depressão e ansiedade (Boelen \& Prigerson, 2007; Prigerson et al., 1999). Portanto, é importante conhecer e estabelecer os critérios para o diagnóstico e posterior intervenção no luto prolongado, assim como capacitar os profissionais da saúde para identificarem indivíduos enlutados em condição de risco que passariam despercebidos se o foco se dirigisse apenas à depressão e à ansiedade (Shear, Frank, Houck, \& Reynolds, 2005).

De acordo com Prigerson et al. (2009), o transtorno do luto prolongado caracteriza-se por sintomas específicos, nomeadamente, intensas saudades e anseio pela pessoa que morreu, descrença ou dificuldade em aceitar a morte, pensamentos intrusivos acerca do falecido, dormência emocional, choque ou confusão, percepção de que a vida é vazia ou sem significado, sentimentos de amargura ou revolta, desconfiança em relação aos outros, sentimento de que parte de si morreu com o falecido, dificuldade em continuar com a própria vida e significativo comprometimento da atividade social ou ocupacional. Holland, Neimeyer, Boelen, \& Prigerson (2009) acrescentam que as reações patológicas distintivas do luto prolongado se definem principalmente pela severidade dos sintomas, que persistem por seis meses ou mais após a perda do ente querido.

A avaliação da severidade das manifestações de luto, por um lado, permite evitar que se rotule como patológica uma reação normal e, por outro, favorece o reconhecimento do sofrimento e da necessidade de garantir suporte para um melhor ajustamento à situação de perda. Para essa avaliação, são necessários instrumentos de medida fiáveis e válidos para a população, que estejam de acordo com a linguagem e as características da população a ser avaliada. Encontramos na literatura internacional alguns instrumentos utilizados para avaliar as manifestações do luto, entre eles o Bereavement Phenomenology Questionnaire (BPQ), o Texas Revised Inventory of Grief (TRIG), o Hogan Grief Reaction Checklist, o Inventory of Complicated Grief (ICG) e o Prolonged Grief Disorder (PG-13). No entanto, até o momento, nenhum desses está validado para a população brasileira.

Com o intuito de preencher essa lacuna no cenário brasileiro, optamos por validar o PG-13 para avaliar o luto prolongado, por ser um instrumento recente, curto, de fácil aplicação e cotação, e que fornece um bom parâmetro sobre as complicações no processo de luto para uma posterior intervenção. O instrumento já foi validado para a população portuguesa (Delalibera, Coelho, \& Barbosa, 2011) e italiana (De Luca et al., 2015) e tem sido utilizado em vários estudos com enlutados (Hudson, Thomas, Trauer, Remedios, \& Clarke, 2011; Thomas, Hudson, Trauer, Remedios, \& Clarke, 2014; Wiese et al., 2010). Nas validações encontradas, a estrutura fatorial do instrumento manteve-se igual ao questionário original e apresentou elevados valores de consistência interna (versão portuguesa 0,932, versão italiana 0,93). Relativamente à incidência do luto prolongado, no estudo italiano que se dedicou a validar o instrumento para a população geral, apenas 6,1\% da amostra preencheu os critérios para o transtorno de luto prolongado. Já no estudo português, $22,5 \%$ da amostra manifestou luto prolon- 
gado, porém a população da pesquisa foi composta por familiares de pacientes em cuidados paliativos.

Portanto, o objetivo do presente estudo foi traduzir, adaptar e validar para a população brasileira o instrumento de avaliação do luto prolongado (prolonged grief disorder - PG-13), elaborado por Prigerson et al. (2008), com o intuito de contribuir para o desenvolvimento de uma medida padrão de avaliação do luto passível de detectar sua incidência e prevalência para auxiliar no tratamento das complicações no processo de luto. Tendo a amostra sido composta pela população geral, e não por uma população clínica, espera-se que a incidência de luto prolongado corresponda à experiência dessa população em luto, e de acordo com as recomendações de Rando et al. (2012) a respeito da inserção do luto complicado no Manual diagnóstico e estatístico de transtornos mentais (DSM-5).

\section{Método}

\section{Participantes}

A amostra deste estudo foi selecionada por conveniência por meio do preenchimento de um questionário on-line. Foi criado um e-mail convite convidando pessoas em luto há pelo menos seis meses a participar do estudo. No e-mail, havia um link que direcionava os interessados à pesquisa presente no site do questionário.

Foram estabelecidos como critérios de inclusão na amostra pessoas que perderam um familiar próximo há pelo menos seis meses e no máximo há cinco anos, ou seja, foram excluídos todos os participantes que estavam em luto há menos de seis meses ou há mais de cinco anos. O questionário foi respondido por 169 pessoas e 54 enlutados foram excluídos por não cumprirem os critérios preestabelecidos.

\section{Instrumentos}

Para caracterizar os participantes, foi utilizado um questionário sócio-demográfico e para avaliar o luto foi utilizado o instrumento de avaliação do luto prolongado - PG13 desenvolvido por Prigerson et al. (2008) com base nos critérios para diagnóstico do transtorno de luto prolongado. O questionário é composto por 13 itens descritivos de um conjunto de sintomas (sentimentos, pensamentos e ações) reativos à perda de um ente significativo, que devem estar presentes pelo menos seis meses após a perda, e estão necessariamente associados a uma disfunção social e/ou funcional. A primeira parte do questionário é constituída por dois itens que avaliam a frequência do sentimento de angústia e de separação; a segunda, por nove itens descritivos de sintomas cognitivos, emocionais e comportamentais; e a última questão é relativa à incapacidade funcional na área social ou ocupacional.

O diagnóstico de luto prolongado implica que se cumpram os seguintes critérios: i) experiência de perda de uma pessoa significativa; ii) angústia de separação (sentir saudades, ausência, intensa dor emocional ou pesar) diariamente ou em grau de extremo sofrimento; iii) sintomas devem estar presentes seis meses após a perda; iv) pelo 
menos cinco dos nove sintomas cognitivos, emocionais e comportamentais devem estar presentes diariamente ou em um grau extremo (por exemplo, evitar o contato com o que faz se lembrar de que a pessoa faleceu; sentir dificuldade em aceitar a perda; sentir que a vida é insatisfatória, vazia ou sem significado desde a perda; não ter a capacidade para confiar nas pessoas; sentir amargura ou raiva em relação à perda); e v) disfunção social, ocupacional ou em outras áreas importantes do funcionamento (por exemplo, responsabilidades domésticas).

Um critério adicional estipula que o transtorno não esteja relacionado a efeitos fisiológicos, consumo de substâncias ou outras condições médicas, e que os sintomas não se explicam melhor por meio da depressão maior, ansiedade generalizada ou transtorno de estresse pós-traumático (Prigerson et al., 2009).

Portanto, para se considerar presente o transtorno de luto prolongado, o entrevistado deve responder aos sintomas das questões 1 e 2 com intensidade diária ou várias vezes ao dia, e os sintomas de angústia de separação devem estar presentes seis meses após a perda. Ou seja, a resposta à pergunta 3 deve ser "sim". A respeito dos sintomas cognitivos, emocionais e comportamentais abordados nas questões de 4 a 12, o sujeito deve assinalar com intensidade diária ou várias vezes ao dia (questões 4 e 5) e bastante ou extremamente (questões 6 a 12) em pelo menos cinco dessas nove questões. Por último, deve-se verificar uma significativa disfunção social e ocupacional, ou seja, a resposta à pergunta 13 deve ser sim.

\section{Procedimentos}

O processo de adaptação e validação do PG-13 para a população brasileira decorreu em várias fases, de acordo com as normas estipuladas por Beaton, Bombardier, Guillemin, \& Ferraz (2000). Em primeiro lugar, foi solicitada a autorização da autora principal para a realização do presente estudo. Seguidamente, realizaram-se duas traduções independentes para a língua portuguesa (do Brasil), efetuadas por duas pessoas bilíngues, sendo uma delas da área de psicologia. Também foi utilizada como base a versão portuguesa do instrumento traduzido e validado por Delalibera et al. (2011).

Depois de resolvidas as discrepâncias existentes entre as duas traduções e elaborada uma versão final, essa versão foi analisada por profissionais da área de psicologia com o objetivo de atingir a equivalência idiomática, semântica e conceitual relativamente ao instrumento original, e garantir a correspondência de significados, a concordância gramatical e a adequação das expressões à cultura brasileira. Após o consenso, a versão final do PG-13 foi pré-testada em grupo de oito enlutados, para verificar a compreensão dos itens e as eventuais dificuldades de interpretação das questões. Em seguida, realizaram-se pequenas adequações apontadas pelos respondentes e a versão final do PG-13 foi concluída. Após a tradução do instrumento, a amostra do estudo foi recolhida por um questionário on-line que ficou disponível em uma página da internet durante o mês de maio de 2014. 
Os dados descritivos da pesquisa foram analisados com o auxílio do programa SPSS (Statistical Package for the Social Sciences), versão 22.0, e a validade fatorial do PG-13 foi avaliada por meio da análise fatorial confirmatória (AFC) realizada com o programa Amos (Analysis of Moment Structures), versão 22.0.

Para a análise fatorial confirmatória foram considerados apenas os 11 itens em que as respostas são obtidas por uma escala tipo Likert. As respostas dos itens 3 e 13 não foram consideradas para os cálculos por serem respostas nominais, dicotômicas (sim/ não) e, portanto, não podem ser utilizadas nesse tipo de análise, e também, por constituírem itens específicos para o diagnóstico clínico do luto prolongado, já que o sujeito obrigatoriamente deve responder positivamente (ou seja, sim) a essas duas perguntas para a confirmação do diagnóstico de luto prolongado.

Avaliou-se a sensibilidade psicométrica por meio das medidas de tendência central e de forma, e a normalidade das variáveis pelos coeficientes de assimetria (sk) e curtose $(\mathrm{ku})$. Os valores de sk são considerados adequados quando inferiores a 3 e os de ku quando inferiores a 7, como descrito por Maroco (2010).

Para o teste de ajuste do modelo proposto, foram analisados os seguintes índices: $\chi^{2} / \mathrm{df}$ (razão qui-quadrado/ graus de liberdade), CFI (comparative fit index), GFI (goodness of fit index) e RMSEA (root mean square error of approximation). De acordo com Maroco (2010), os índices de adequação do modelo são considerados satisfatórios para os valores de $\chi^{2} / \mathrm{df}$ inferiores a $3, \mathrm{CFI}$ e GFI superiores a 0,90 e de RMSEA inferiores a 0,10 .

A validade convergente foi estimada pela variância extraída média (VEM), e de acordo com Maroco (2010), os valores de $\operatorname{VEM~} j \geq 0,5$ indicam validade convergente. A consistência interna foi avaliada pelo coeficiente alfa de Cronbach $(\alpha)$ e pela confiabilidade compósita (CC) e os valores de $\mathrm{CC} j \geq 0,7$ indicam confiabilidade de construto do instrumento.

A validade fatorial foi avaliada pelos pesos fatoriais estandardizados e, segundo Maroco (2010), se os pesos fatoriais de todos os itens forem iguais ou superiores a 0,5 , o fator apresenta validade fatorial e a fiabilidade individual dos itens $\left(R^{2}\right)$ também deve ser igual ou superior 0,25 para ser considerada apropriada.

Este estudo integra uma pesquisa mais ampla aprovada pelo Comitê de Ética em Pesquisa do Instituto Universitário de Lisboa (Ispa), Portugal, e pelo Comitê de Ética em Pesquisa do Hospital Santa Marcelina, São Paulo, Brasil (Parecer n. 687.742).

\section{Resultados}

\section{Tabela I. Caracterização sociodemográfica da população}

\begin{tabular}{lcc} 
& Participantes $(\mathrm{N}=115)$ & Familiares falecidos \\
\hline Idade média (desvio padrão) & $41,32(\mathrm{DP}=14,43)$ & $65,12(\mathrm{DP}=21,39)$ \\
Amplitude & $19-80$ & $23-93$ \\
\hline
\end{tabular}


Tabela I. Caracterização sociodemográfica da população (conclusão)

\begin{tabular}{lcc}
\hline & Participantes $(\mathrm{N}=$ II 5$)$ & Familiares falecidos \\
\hline Gênero N (\%) & $27(23,5)$ & $65(57)$ \\
Masculino & $88(76,5)$ & $49(43)$ \\
Feminino & & \\
\hline Estado Civil N (\%) & $38(33)$ & $19(16,5)$ \\
Solteiro & $58(50,4)$ & $60(52,2)$ \\
Casado/ União estável & $4(3,6)$ & $30(26,1)$ \\
Viúvo & $15(13)$ & $6(5,2)$ \\
Separado/ Divorciado & & \\
\hline Escolaridade N (\%) & $14(12,3)$ & \\
Ensino médio & $40(35,1)$ & \\
Ensino superior & $42(36,8)$ & \\
Pós-Graduação & $15(13,2)$ & \\
Mestrado & $3(2,6)$ & \\
Doutorado & & \\
\hline Parentesco N (\%) & $3(2,6)$ & \\
Cônjuge & $42(36,5)$ & \\
Filho(a) & $14(12,2)$ & \\
Pai/Mãe & $30(26,1)$ & \\
Neto(a) & $10(8,7)$ & \\
Irmão(a) & $11(9,5)$ & \\
Sobrinho(a) & $5(4,4)$ & \\
Outros familiares & & \\
\hline
\end{tabular}

Fonte: Elaborado pelos autores.

A população do estudo foi constituída por 115 familiares enlutados. Desses familiares, $76,5 \%$ eram do sexo feminino, 50,4\% casados, média de idade de 41,32 anos e $87,7 \%$ dos participantes haviam concluído pelo menos o ensino superior (Tabela 1). Relativamente ao grau de parentesco, a maioria $(36,5 \%)$ dos participantes era filho(a) do falecido, e a principal causa de morte $(27,8 \%)$ foi por doença oncológica, apesar da causa de morte ter variado bastante entre doenças crônicas, como: doenças cardíacas $(19,3 \%)$, respiratórias $(11,3 \%)$, renais $(5,2 \%)$ e morte repentina $(13 \%)$, morte violenta (assassinato e suicídio - 8,7\%), acidentes de trânsito (7\%) e outras menos prevalentes (totalizando 7,7\%).

A sensibilidade psicométrica avaliada pela assimetria (sk) e curtose (ku) apresentou para todos os itens do instrumento valores de assimetria superior a 3 e de curtose superior a 7, o que indica que não existem problemas na distribuição normal e na sensibilidade dos itens.

Uma vez verificados os pressupostos de aplicação, procedeu-se a análise fatorial confirmatória à estrutura fatorial original do PG-13 proposta pelos autores do instrumento, no sentido de avaliar a validade e confiabilidade da escala. Na figura 1, é apre- 
sentado o modelo de medida inicial com os pesos fatoriais e os erros para os onze componentes analisados. Como é possível observar, todos os pesos fatoriais são superiores a 0,5 , o que significa que todos os itens do instrumento contribuem para a formação do fator.

\section{Figura I. Estrutura fatorial do modelo inicial e do modelo reestruturado do PG-I3.}
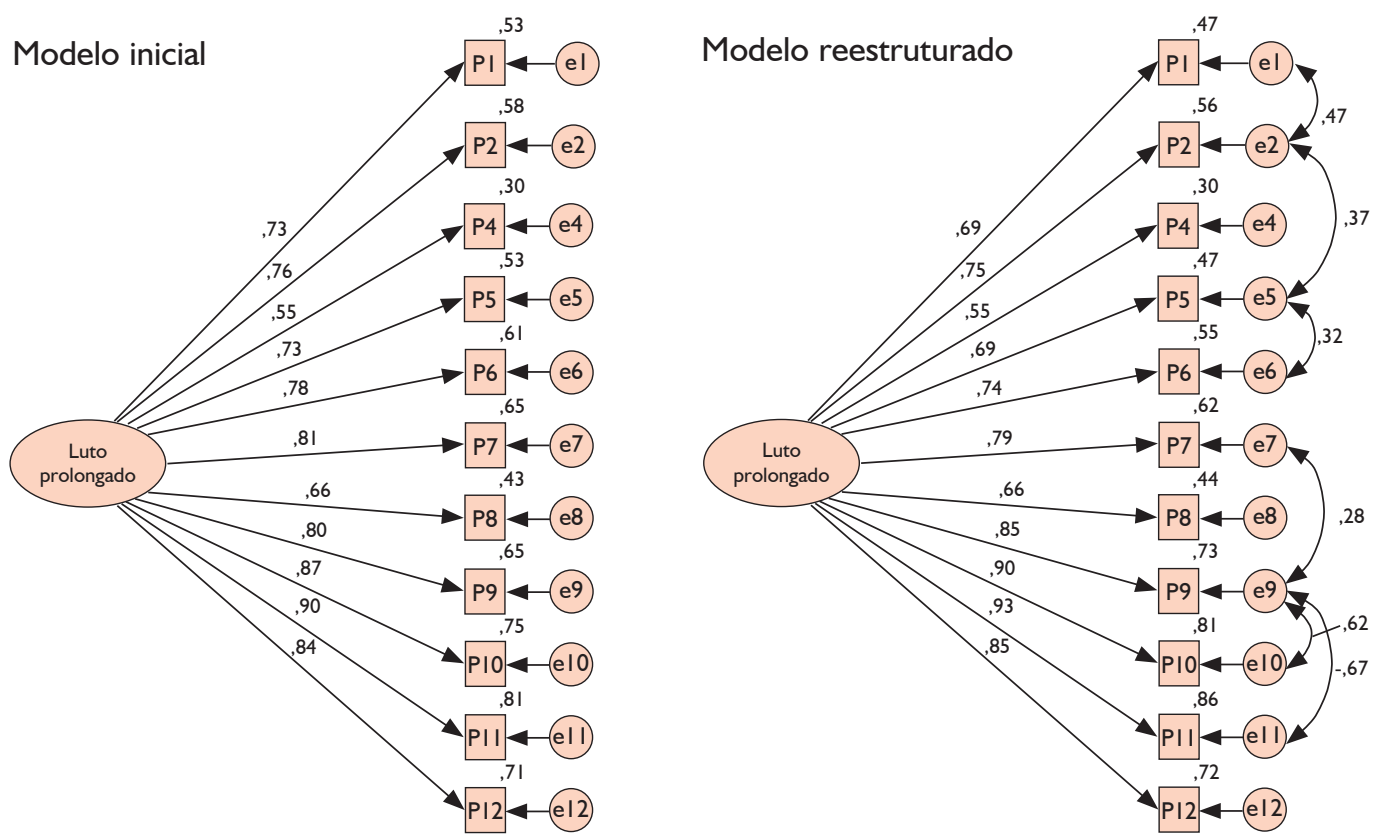

Fonte: Elaborado pelos autores.

Tabela 2. Índices de adequação dos modelos testados.

\begin{tabular}{ccc}
\hline Índice & Modelo inicial & Modelo reestruturado \\
\hline$\chi^{2} / \mathrm{df}$ & 4,487 & 1,915 \\
CFI & 0,848 & 0,965 \\
GFI & 0,724 & 0,891 \\
RMSEA & 0,175 & 0,090 \\
\hline
\end{tabular}

Fonte: Elaborado pelos autores.

No entanto, os índices de adequação do modelo inicial não estão muito próximos do satisfatório, o que pode ser observado na Tabela 2. Devido aos valores dos índices estarem afastados do aceitável, foi realizada gradualmente uma reestruturação do 
modelo de acordo com as especificações da análise dos índices de modificação e as covariâncias entre os erros dos itens 1 e 2, 2 e 5, 7 e 9, 9 e 11, 9 e 10, 5 e 6 (Figura 1), melhorando assim, os valores dos índices de adequação em relação ao modelo inicial e aproximando os valores dos índices do satisfatório (Tabela 2), classificando assim o modelo como adequado.

Tabela 3. Itens analisados do PG-13 e respectivos pesos fatoriais

\begin{tabular}{|c|c|c|c|}
\hline Item & Peso fatorial & VEM & $\mathrm{R}^{2}$ \\
\hline $\begin{array}{l}\text { I. No último mês, com que frequência sentiu saudades ou a } \\
\text { ausência da pessoa que perdeu? }\end{array}$ & 0,686 & 0,593 & 0,470 \\
\hline $\begin{array}{l}\text { 2. No último mês, com que frequência sentiu intensa dor } \\
\text { emocional, tristeza/pesar ou episódios de angústia relacionados } \\
\text { com a relação perdida? }\end{array}$ & 0,747 & & 0,558 \\
\hline $\begin{array}{l}\text { 4. No último mês, com que frequência tentou evitar contato com } \\
\text { o que lhe faz lembrar que a pessoa realmente faleceu? }\end{array}$ & 0,547 & & 0,299 \\
\hline $\begin{array}{l}\text { 5. No último mês, quantas vezes se sentiu atordoado/a, chocado/a } \\
\text { ou confuso pela sua perda? }\end{array}$ & 0,686 & & 0,470 \\
\hline $\begin{array}{l}\text { 6. Sente-se confuso/a quanto ao seu papel na vida ou sente que } \\
\text { não sabe quem é desde a sua perda? }\end{array}$ & 0,744 & & 0,553 \\
\hline 7. Tem tido dificuldade em aceitar a perda? & 0,789 & & 0,622 \\
\hline 8. Tem tido dificuldade em confiar nos outros desde a sua perda? & 0,665 & & 0,442 \\
\hline 9. Sente amargura pela sua perda? & 0,852 & & 0,726 \\
\hline $\begin{array}{l}\text { 10. Sente que continuar com a sua vida seria difícil para você } \\
\text { agora? }\end{array}$ & 0,901 & & 0,812 \\
\hline II. Sente-se emocionalmente dormente desde a sua perda? & 0,926 & & 0,858 \\
\hline $\begin{array}{l}\text { 12. Sente que a sua vida é insatisfatória, vazia ou sem significado } \\
\text { desde a sua perda? }\end{array}$ & 0,848 & & 0,718 \\
\hline
\end{tabular}

Fonte: Elaborado pelos autores.

Os itens do modelo reestruturado do PG-13 apresentaram pesos fatoriais elevados $(\lambda \geq 0,5)$ e estatisticamente significativos ( $p<0,001)$, com confiabilidades individuais adequadas ( $R 2 \geq 0,25$ ) (Tabela 3 ), indicando que cada um dos onze itens analisados do PG-13 compreende um marcador importante no construto do luto prolongado.

O índice de consistência interna, avaliado tanto pelo alfa de Cronbach quanto pela confiabilidade compósita, foi de 0,940, e a validade convergente avaliada pela VEM foi de 0,593 , pelo que se pode concluir que o instrumento apresenta confiabilidade de construto, e também validade fatorial e convergente. 
A incidência de luto prolongado na população do estudo foi de $10,43 \%$, o tempo de luto variou entre seis meses e cinco anos com uma média de 29,29 meses de luto $(D P=16,99)$.

\section{Discussão}

O presente estudo teve como principal objetivo realizar a análise fatorial confirmatória da versão brasileira do instrumento de avaliação do luto prolongado PG-13 e utilizou a estrutura unidimensional do instrumento de acordo com os autores da versão original.

A AFC demonstrou que todos os itens do PG-13 apresentaram pesos fatoriais superiores a 0,5 , portanto, não foi preciso retirar nenhum item do instrumento. Já o modelo inicial não produziu índices de adequação satisfatórios e, por isso, foi necessária uma reestruturação do modelo. A covariância entre os itens 1 e 2 pode ter ocorrido devido a ambos os itens estarem relacionados com a angústia de separação (sentir saudades, ausência, intensa dor emocional ou pesar). No caso dos itens 5 e 6 , como pode ser observado na Tabela 3, ambos descrevem sentimentos de confusão, e os itens 7 e 9 avaliam de perspectivas diferentes a dificuldade em lidar com a perda e, provavelmente, os respondentes podem ter considerado que as perguntas eram similares. Com relação aos itens 9 e 10 e 9 e 11, que são referentes a sintomas emocionais, a covariância é negativa, o que não faz muito sentido, pois quanto mais amargurado o enlutado estivesse, era suposto que mais dificuldade teria para continuar com a sua vida, e não o contrário, o que nos faz pensar se os participantes compreenderam e responderam de maneira adequada a essas perguntas.

A consistência interna, importante medida de confiabilidade avaliada tanto pelo alfa de Cronbach quanto pela confiabilidade compósita, apresentou valores elevados, superiores a 0,90, o que confirma a confiabilidade de construto do PG-13, e de acordo com resultados da VEM também podemos concluir que o instrumento tem validade convergente.

Com relação à porcentagem de luto prolongado na população do estudo $(10,43 \%)$, a incidência está de acordo com os parâmetros reportados na literatura internacional - 10 a $20 \%$ da população enlutada - (Boelen \& Prigerson, 2007; Prigerson et al., 2008; Prigerson, 2004), e um pouco superior aos $6,1 \%$ do estudo de validação italiano, que também foi realizado com a população geral (De Luca et al., 2015). No entanto, é muito inferior quando comparado com os estudos realizados com populações específicas de familiares enlutados de pacientes que foram acompanhados em cuidados paliativos, como é o caso do estudo de Delalibera et al. (2011), realizado em Portugal, em que 22,5\% dos participantes cumpriam os critérios para o diagnóstico de luto prolongado e do estudo de Wiese et al. (2010), realizado na Alemanha, em que a incidência foi de 30\%. Essa diferença pode ser explicada porque no presente estudo não se delimitou o perfil dos enlutados em relação à causa de morte do familiar falecido e o tempo de luto do participante que respondeu ao questionário foi maior do que nas outras investigações. 
Estudos (Neimeyer \& Hogan, 2001; Neimeyer, Hogan, \& Lauri, 2008) ressaltam a importância do cuidado na mensuração do luto, quanto aos aspectos culturais e a natureza do fenômeno em si. Destacam o risco de se mensurar fenômenos não bem-conceituados e com uso ingênuo dos recursos psicométricos. Neste estudo, esses cuidados foram considerados e permitiram a discussão dos resultados de maneira metodologicamente fundamentada.

\section{Conclusão}

De acordo com os resultados obtidos por meio da AFC realizada, podemos concluir que o instrumento apresenta validade convergente e confiabilidade de construto, portanto, o PG-13 é um instrumento adequado para o diagnóstico e a avaliação da população enlutada brasileira. Contudo, deve-se ter cuidado com a generalização dos resultados, uma vez que a amostra foi obtida por conveniência e outros estudos devem ser realizados com populações específicas para se verificar a incidência do luto prolongado nessas populações.

Como limitações do estudo, podemos apontar que a amostra foi escolhida por conveniência e não é representativa da população geral brasileira. Tiveram acesso e puderam responder ao questionário apenas pessoas com acesso a computadores e internet, consequentemente, o nível de escolaridade da amostra é superior ao da população geral brasileira. No entanto, é importante a realização de outros estudos com uma amostra alargada e/ou com outras populações mais específicas. Como sugestões para futuras investigações, recomendamos desenvolver uma avaliação da morbidade física e mental e de outros fatores de vulnerabilidade associados ao transtorno de luto prolongado para melhor compreensão da incidência e sintomatologia, e posterior encaminhamento para acompanhamento psicológico adequado.

\section{Referências}

Beaton, D. E., Bombardier, C., Guillemin, F., \& Ferraz, M. B. (2000). Guidelines for the process of cross-cultural adaptation of self-report measures. Spine, 25(24), 3186-3191. doi: 10.1097/00007632-200012150-00014.

Boelen, P. A., \& Prigerson, H. G. (2007). The influence of symptoms of prolonged grief disorder, depression, and anxiety on quality of life among bereaved adults: a prospective study. European Archives of Psychiatry and Clinical Neuroscience, 257(8), 444-52. doi: 10.1007/s00406-007-0744-0.

Boerner, K., Mancini, A.D., \& Bonanno, G. (2013). On the nature and prevalence of uncomplicated and complicated patterns of grief. In M. Stroebe, H. Schut, \& J. Van den Bout. Complicated grief. Scientific foundations for health care professionals (pp. 55-67). Nova York: Routledge. 
De Luca, M. L., Tineri, M., Zaccarello, G., Grossi, G., Altomonte, A., Slavic, E., ... Greco, R. (2015). Adattamento e validazione del questionario "PG-13" Prolonged Grief nel contesto italiano. Rivista Italiana di Cure Palliative, 17(2), 1-9.

Delalibera, M., Coelho, A., \& Barbosa, A. (2011). Validação do instrumento de avaliação do luto prolongado para a população portuguesa. Acta Médica Portugesa, 24(6), 935-942.

Holland, J. M., Neimeyer, R. A., Boelen, P. A., \& Prigerson, H. G. (2009). The underlying structure of grief: a taxometric investigation of prolonged and normal reactions to loss. Journal of Psychopathology and Behavioral Assessment, 31(3), 190-201. doi: 10.1007/s10862-008-9113-1.

Hudson, P. L., Thomas, K., Trauer, T., Remedios, C., \& Clarke, D. (2011). Psychological and social profile of family caregivers on commencement of palliative care. Journal of Pain and Symptom Management, 41(3), 522-534. doi: 10.1016/j.jpainsymman.2010.05.006.

Maroco, J. (2010). Análise de equações estruturais: fundamentos teóricos, software \& aplicações. Pero Pinheiro: ReportNumber, Lda.

Neimeyer, R., \& Hogan, N. (2001). Quantitative or Qualitative? Measurement issues in the study of grief. In M. Stroebe, R. Hansson, W. Stroebe, \& H. Schut (Ed.). Handbook of bereavement research, consequences, coping and care (pp. 89-118). Washington: American Psychological Association Press. doi: 10.1037/ 10436-004.

Neimeyer, R. A., Hogan, N. S., \& Laurie. A. (2008). The measurement of grief: psychometric considerations in the assessment of reactions to bereavement. In M. Stroebe, R. Hansson, H. Schut, \& W. Stroebe (Ed.). Handbook of bereavement research and practice: Advances in theory and intervention (pp.133-161). Washington: American Psychological Association Press. doi: 10.1037/14498-007.

Parkes, C. M. (1998). Luto: estudos sobre a perda na vida adulta. São Paulo: Summus.

Prigerson, H. (2004). Complicated grief: when the path of adjustment leads to a dead-end. Bereavement Care, 23(3), 38-40. doi: 10.1080/02682620408657612.

Prigerson, H. G., Maciejewski, P. K., Reynolds, C. F., Bierhals, A. J., Newsom, J. T., Fasiczka, A., ... Miller, M. (1995). Inventory of complicated grief: a scale to measure maladaptive symptoms of loss. Psychiatry Research, 59(1-2), 65-79. doi: 10.1016/0165-1781(95)02757-2.

Prigerson, H., Bierhals, A., Kasl, S., Reynolds, C. F., Shear, M. K., Day, N., ... Jacobs, S. (1997). Traumatic grief as a risk factor for mental and physical morbidity. American Journal of Psychiatry, 154(5), 616-623. doi: 10.1176/ajp.154.5.616.

Prigerson, H. G., Shear, M. K., Jacobs, S. C., Reynolds, C. F., Maciejewski, P. K., Davidson, J. R., ... Zisook, S. (1999). Consensus criteria for traumatic grief. A preliminary 
empirical test. The British Journal of Psychiatry, 174(1), 67-73. doi: 10.1192/bjp. 174.1.67.

Prigerson, H., Vanderwerker, L., \& Maciejewski, P. (2008). Prolonged grief disorder: a case for inclusion in DSM. In M. Stroebe, R. Hansson, H. Schut, \& W. Stroebe. (Eds.). Handbook of bereavement research and practice: advances in theory and intervention (pp. 165-186). Washington: American Psychological Association Press. doi: 10.1037/14498-000.

Prigerson, H. G., Horowitz, M. J., Jacobs, S. C., Parkes, C. M., Aslan, M., Goodkin, K., ... Maciejewski, P. K. (2009). Prolonged grief disorder: psychometric validation of criteria proposed for DSM-V and ICD-11. PLOS Medicine, 6(8), 1-12. doi: 10.1371/journal.pmed.1000121.

Rando, T. A., Doka, K. J., Fleming, S., Franco, M. H., Lobb, E. A., Parkes, C. M., \& Steele, R. (2012). A call to the field: complicated grief in the DSM-5. OMEGA-Journal of Death and Dying, 65(4), 251-255. doi: 10.2190/OM.65.4.a

Rando, T. A. (2013). On achieving clarity regarding complicated grief: lessons from clinical practice. In: M. Stroebe, H. Schut, \& J. Van den Bout. (Eds.). Complicated grief. scientific foundations for health care professionals (pp. 40-54). New York: Routledge.

Shear, K., Frank, E., Houck, P. R., \& Reynolds, C. F. (2005). Treatment of complicated grief: a randomized controlled trial. JAMA, 293(21), 2601-2608. doi: 10.1001/jama.293.21.2601.

Thomas, K., Hudson, P., Trauer, T., Remedios, C., \& Clarke, D. (2014). Risk factors for developing prolonged grief during bereavement in family careers of cancer patients in palliative care: a longitudinal study. Journal of Pain and Symptom Management, 47(3), 531-541. doi: 10.1016/j.jpainsymman.2013.05.022.

Wiese, C. H. R., Morgenthal, H. C., Bartels, U. E., Vossen-Wellmann, A., Graf, B. M., \& Hanekop, G. G. (2010). Post-mortal bereavement of family caregivers in Germany: a prospective interview-based investigation. Wiener Klinische Wochenschrift, 122(13-14), 384-389. doi: 10.1007/s00508-010-1396-z.

Submissão: 8.12 .2015

Aceitação: 3.10 .2016 